\title{
CANCERS AFFECTING WOMEN IN MALAYSIA
}

\author{
Maizura Binti Musa, Fariza Bt. Mohd Yusof, Md. Harun-Or-Rashid, Junichi Sakamoto \\ Young Leaders' Program in Healthcare Administration, Nagoya University Graduate School of Medicine
}

\begin{abstract}
Cancer incidence in women has been slightly higher than men in Malaysia, related somewhat to the lifestyles and socioeconomic development of the country. It is one of the debilitating health problems affecting women. Of the 10 most common cancers affecting women of Malaysia, breast cancer is the biggest threat, followed by colorectal and cervical cancer according to the latest available national report in 2006. Malaysia offers a variety of treatment centers and choice of treatments; some are guided by the availability of national guidelines, such as that for breast and cervical cancers. Research has actively been conducted to find out the best and future approach on screening, diagnosis and treatment for cancers affecting women. This is made possible with the government taking the lead and the important works which are highly supported by the relevant non-governmental bodies and the public. Improvement is still needed for increasing coverage of screening and public awareness on cancer prevention.
\end{abstract}

Key Words: cancer, incidence, women, Malaysia.

(Received June 15, 2011; Accepted June 24, 2011)

\section{Introduction}

Malaysia is a South East Asian country which is comprised of two parts, namely the Peninsular Malaysia and the East Malaysia, which shares part of the Borneo Island with Brunei Darussalam and Indonesia. The country has a population of $27,565,821$ which comprises $14,112,667$ males and $13,453,154$ females $^{1)}$. It is also known as a multi-ethnic country, of which its population is characterized by three major ethnic groups: the Malays (62\%), Chinese (27\%) and Indians (9\%). The country's increasing socioeconomic development since the 1980s, leading to changes in lifestyles and the increase in survival age due to better health outcomes were thought, in return to have led to the rise in the incidence of cancer. In Peninsular Malaysia in the year 2006, a total of 21,773 cancer cases were diagnosed, comprised of 9,974 males and 11,799 females. The age standardized incidence rate (ASR) for all cancers regardless of sex then was 131.3 per 100,000 and the five most common cancers among population of Peninsular Malaysia were breast, colorectal, lung, cervical and nasopharyngeal cancers ${ }^{2}$.

There has been much progress achieved in Malaysia that the gender gap is seen to be narrowing in the past few decades. Women of Malaysia currently have almost equal contribution to men in the socioeconomic develop-

Corresponding Author: Maizura binti Musa, Young Leaders' Program (YLP) in Healthcare Administration, Nagoya University Graduate School of Medicine, 65 Tsurumai-Cho, Showa-ku, Nagoya, 466-8550 Japan. TEL/FAX: +81-52-744-2444, e-mail:maizuramusa@yahoo.com ment of the country, where many have been known to have held important and high professional positions in both public and private sectors. Hence they are also considered assets to the country, and their health and wellbeing are important in order for the country to continue to bloom and grow. Due to the interest on the health status of women in Malaysia, we would like to explore the facts on cancers, especially the most common cancers affecting Malaysian women including the relevant statistics, current situation and the development in prevention, diagnosis and treatment approach.

\section{Materials and Methods}

This paper was prepared based on the data of cancer incidence and mortality from multiple sources. The main source of cancer statistical data was from the National Cancer Registry (NCR) report from 2002 to 2006. The NCR was the first effort of central coordination for the purpose of obtaining accurate data for cancer in Malaysia. It was initiated by the Ministry of Health $(\mathrm{MoH})$ of Malaysia and involved up to 220 participating centres (both public and private) in Peninsular Malaysia as sources of data annually. Additional data from individual or institutional research and World Health Organization (WHO) sources were also used where the relevant data from $\mathrm{MoH}$ were not available.

\section{Cancer incidence and mortality}

The NCR has produced four official reports since it 
first started in the year 2002. The male to female ratio of cancers incidence are around $1: 1.2$, and this condition of slightly higher female incidence were the same throughout the years from year 2002 to 2006, where median age at diagnosis of cancer in women was reported around 53 years $^{2-5)}$. Cancer incidence in females by age is as shown in Table 1 and the 10 most common cancers affecting women in general are as shown in Fig. 1 .

Breast cancer has been noted since the past 10 years as the most common cancer affecting Malaysian women. It comprised $31 \%$ of all cancers affecting women, and 1 in 20 women have the chance of developing it in their lifetime $^{6}$. This cancer is also considered a debilitating health threat to women, evidenced by the report of 124.87 age-standardized disability-adjusted life years (DALYs) due to breast cancer according to WHO Global Health Data Repository in 2004. Since 2003, the age standardized incidence rate (ASR) was reported as high-

Table 1. Cancer incidence in females per 100,000 population (CR) by age in Peninsular Malaysia, 2006

\begin{tabular}{|c|r|r|r|}
\hline Age in years & No & \multicolumn{1}{|c|}{ Percentage } & \multicolumn{1}{c|}{ CR } \\
\hline $0-9$ & 52 & 0.4 & 2.6 \\
\hline $10-19$ & 199 & 1.7 & 10.1 \\
\hline $20-29$ & 523 & 4.4 & 31.0 \\
\hline $30-39$ & 1215 & 10.3 & 88.7 \\
\hline $40-49$ & 2552 & 21.6 & 213.1 \\
\hline $50-59$ & 3193 & 27.1 & 380.9 \\
\hline $60-69$ & 2243 & 19.0 & 484.8 \\
\hline $70+$ & 1822 & 15.4 & 588.1 \\
\hline
\end{tabular}

er among the Chinese and Indians in comparison to the Malays. This trend continued in the NCR report in 2006, where the incidence of breast cancer was highest among Chinese, followed by Indians and Malays (Table 2). The age pattern showed a peak ASR at the 50-59 age groups. It also constitutes $3.1 \%$ deaths in women as shown in the report by Department of Statistics Malaysia in 2007.

Colorectal cancer was the second most common cancer among females in Peninsular Malaysia with ASR of 15.4 per 100,000 populations in the 2006 NCR report. The incidence in females was lower than males. However, women of more than 70 years of age had higher incidence of colorectal cancer than breast cancer.

Cancer of the cervix was the third most common cancer among women. A world report in 2007 reported that the incidence of cervical cancer was 1,492 cases with ASR of 15.7 per 100,000 population and mortality cases of 766 with ASR of 8.4 per 100,000 population ${ }^{7}$.

Table 2. Cancer incidence and age-standardized incidence rate (ASR) per 100,000 populations by ethnicity of women, Peninsular Malaysia, 2006

\begin{tabular}{|c|r|r|r|}
\hline Ethnic group & \multicolumn{1}{|c|}{ No } & \multicolumn{1}{c|}{ percentage } & \multicolumn{1}{c|}{ ASR } \\
\hline Malay & 4753 & 46.4 & 96.4 \\
\hline Chinese & 4422 & 43.2 & 151.5 \\
\hline Indian & 1065 & 10.4 & 134.7 \\
\hline
\end{tabular}

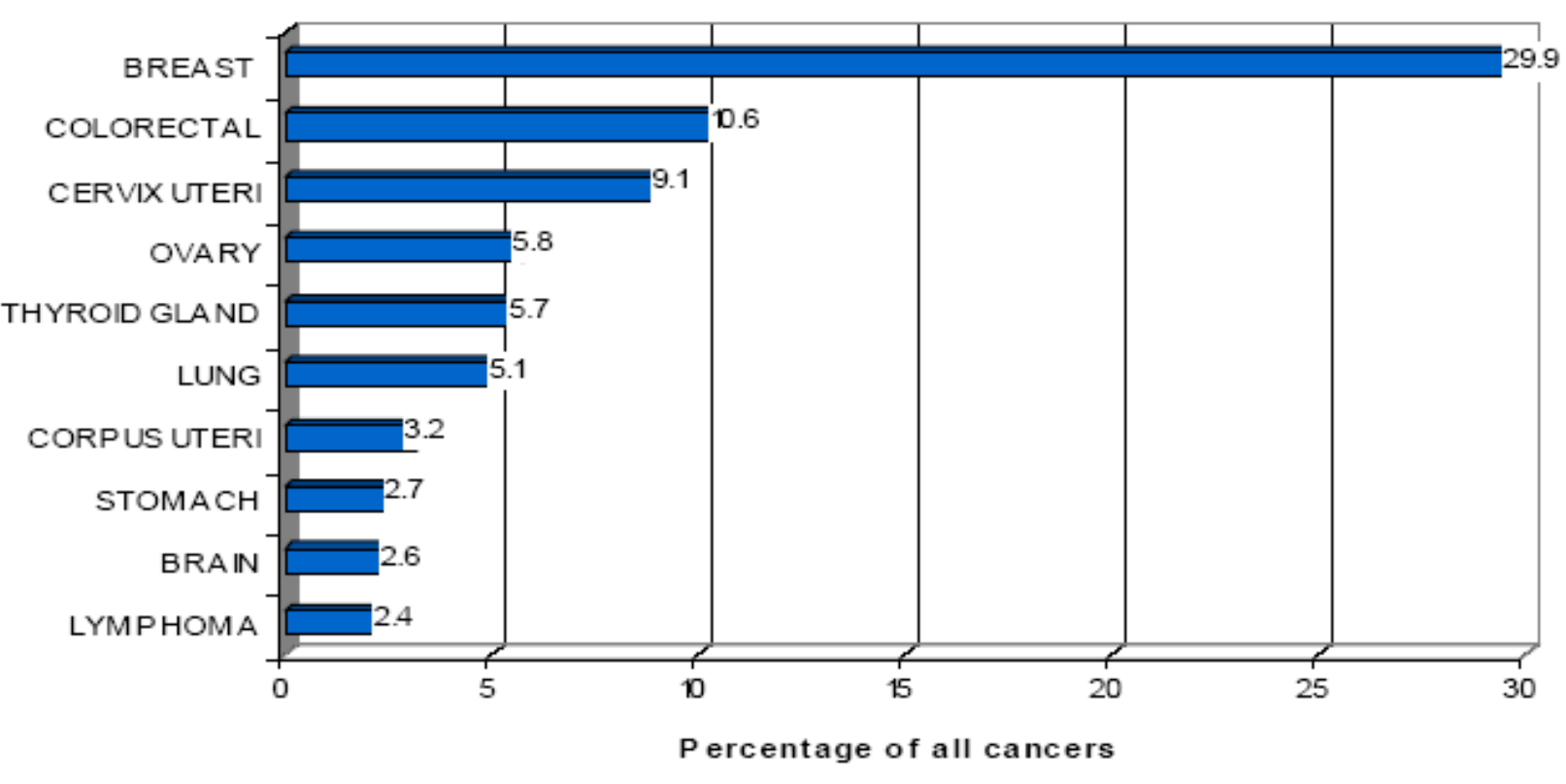

Fig. 1 The 10 most frequent cancers in females, Peninsular Malaysia 2006 (Malaysian Cancer Statistics - Data and figure, Peninsular Malaysia 2006) 
According to NCR 2006 report, cervical cancer incidence rate increased with age after 30 years and has its peak at ages 60-69 years.

Apart from the above, the incidence of cancer can be further divided according to age categories, namely the women of young-age group (15-49 years old) and oldage group (50 years old and above). This age division is important because most common cancers were different among these age groups. In the young-age group, cancers of the ovary, uterus, thyroid gland and leukemia came next after breast and cervical cancers. In the old-age group, colorectal came second after breast cancer, followed by cervical, uterus, and lung cancers.

\section{Risk factors for cancer}

As for other types of illnesses, studies have pointed out some factors leading to the incidence of cancer affecting Malaysian women.

\section{Ethnic groups}

As we could see from the above statistics, there was a difference in ethnicity seen with the incidence of cancers in women. Though breast cancer remains the most common cancer among all three main ethnic groups, the incidence was higher among Chinese and Indians than Malays. The reason is based on the fact that Malay women usually get married at earlier age, have more children and breast feed their children longer than women of other ethnic groups. Having first child early, more children and longer period of breast feeding are well-known protective factors against breast cancer. Though the incidence is higher in Chinese women, a study showed that patients of Malay ethnic group presented with breast cancer at more advanced stage and the cancerous lesions tend to be much larger than others ${ }^{8)}$.

From the NCR reports, Chinese women were also found to have the highest incidence of cervical cancer followed by Indians and Malays. In addition, Malay women were found to be more prone to cancers of the colon, cervix, leukemia and ovaries. Chinese women have high incidence of cancers of the colon, cervix, lung and stomach, while Indian women had higher chance of getting cancers of the cervix, mouth, colon and ovaries.

\section{Lifestyle and nutrition}

The percentage of women smokers is relatively low in Malaysia. As of 2006, 2.6\% women were smokers, this is not considered the main factor for cervical cancer risk. The National Health Morbidity Survey data reported that, $20.7 \%$ adults were overweight and $5.8 \%$ were obese ( $0.3 \%$ of whom had BMI values of $>40.0 \mathrm{kgm}-2)$; where the prevalence of obesity was greater in women than in men $^{9)}$. Obesity has been known to be associated with an increased risk of developing breast cancer with higher mortality rate.

Dietary differences between the different racial groups could be an important factor for development of cancer. It has been suggested socioeconomic status is linked to the diet during early adulthood consisting of dairy products and animal fats, making a person at risk of developing cancer especially the breast cancer. In terms of socioeconomic status, the Chinese are socioeconomically more developed than the other races, and this is thought as the reason why they have higher breast cancer incidence.

\section{Genetic factors}

A few studies have shown that genetic factors leading to cancers have been identified. Family history of breast cancer is an independent risk factor, where the risk is higher among young first degree relatives. If there is a sister with breast cancer, there is higher risk of other siblings to have breast cancer.

It has been well-established that hereditary breast cancer is primarily due to germ line mutations in one of two breast cancer susceptibility genes, BRCA1 and BRCA2. A cohort of 37 individuals with breast cancer disease showed the characteristic of a spectrum of BRCA mutations. BRCA1 and BRCA2 sequence alterations were reported, which represent a prevalence of $2.7 \%$ and $5.4 \%$ respectively, consistent with other studies in Asian countries ${ }^{10)}$. There is also an association between the TP53binding protein 1 (TP53BP1) genetic polymorphism and breast cancer risk as well as clinicopathological characteristics as seen in 387 breast cancer patients. A study concluded that Glu353Asp variant of TP53BP1 might be associated with tumor aggressiveness ${ }^{11)}$.

\section{Other risk factors}

The Human Papillomavirus (HPV) infection has been noted as the cause of nearly all cases of cervical cancer. However, data on HPV prevalence in the general population of Malaysia is not yet available.

\section{Cancer prevention, screening and diagnosis}

\section{Breast cancer screening}

Breast self-examination (BSE) is well-known as a relatively cheap and effective method of detecting breast lump. The MoH has introduced BSE at primary care level, mostly targeting those women who present themselves for family planning at government clinics. However, a recent study of 60 women who were breast cancer survivors identified that $80 \%$ of them detected their breast lumps by self-detection, despite in fact, $85 \%$ of them never knew about BSE beforehand. This study has also shown that proper education led to an increase of monthly BSE practices from $17 \%$ (at pre-test) to $67 \%$ (at post-test) for these women ${ }^{12)}$. This suggest that aware- 
ness program and improvement in education about BSE is still needed to improve the current situation.

The use of mammography as screening method is certain but studies shown that this need to be targeted to certain types of people especially those with family history of breast cancer. Screening women of high risk should be done from the age of 30 years. Both Magnetic Resonance Imaging and mammography should be used as it is more effective than mammography alone. In addition, it was noted that higher breast density finding from mammography is associated with higher risk ranges from two times in scattered fibro glandular density to four times in an extremely dense breast ${ }^{13)}$.

\section{Cervical cancer screening}

The screening program for cervical cancer using Pap smear was first initiated in 1969, initially opportunistic screening which later extended to include all women undergoing family planning. In 2003, a National Guidelines on Pap Smear Screening produced recommended that all sexually active women aged 20-65 years should attend screening annually for two consecutive years. If both smears were normal, screening could continue every 3 years. The $\mathrm{MoH}$ had even offered free Pap smear tests to women attending public healthcare facilities. However, WHO Health Surveys in 2001-2002 obtained that Pap smear coverage was only $23 \%$ of women. The highest uptake was among women aged $30-39$ years $(36.6 \%)^{14)}$.

Similarly, a study on 221 women aged 25 to 85 years old, diagnosed with cervical cancer from the year 2000 to 2006 also pointed out that a large number of them had not had a Pap smear test within 3 years preceding cancer development. The percentage of patients who had had the test done was below $12 \%$. The main reasons cited for not having had a Pap smear test done were "Never heard about it" (36.2\%), "Shy" (10.4\%), "Afraid to do it" (13.1\%), "Think the test is not important" (8.1\%), and "No encouragement from family" $(4.5 \%)^{15)}$. In addition, another study found out that Indian women were the least likely to have had a Pap smear and knew the reasons why screening was necessary. Malay women on the other hand, were less likely than Chinese women to have received a Pap smear and more likely to report embarrassment as the reason. Urban women were less likely than rural women to undergo Pap smear test and more likely to state lack of time as the reason ${ }^{16)}$. These recent studies suggest that interventions targeted to certain groups of women at risk may be a better solution in the attempt to increase screening rates in Malaysia.

\section{Other screening}

Until now there is no active national screening program for other types of cancers frequently affecting women including colorectal cancer. Mass screening at a national level cannot be recommended for any individu- als, therefore the need to carefully study the population at risk. A detailed cancer registry of all patients with colorectal cancer in Malaysia just set up by $\mathrm{MoH}$ in 2008 will hopefully guide the decision on future direction on screening method.

\section{Cancer prevention}

A National Immunization Technical Committee under the Disease Control Division of $\mathrm{MoH}$ was given the responsibility to study and make recommendations on the role of the HPV vaccine in Malaysia in the attempt to prevent cervical cancer. The Malaysia Drug Authority had also approved the use of the quadrivalent HPV vaccine (Gardasil ${ }^{\circledR}$, Merck \& Co., Inc., Whitehouse Station, NJ, USA) in October 2006. The first introduction of the vaccine nationwide has started in year 2010, targeting female school children aged 13 years and more ${ }^{17)}$.

\section{Cancer treatment}

\section{Cancer treatment and cancer specialists' centers}

There are few cancer centers both in public and private healthcare sectors. It is known that there are at least 82 government hospitals that can treat cancer patients. Some of the well-known ones are Hospital Kuala Lumpur, Hospital Umum Sarawak and Hospital Sultan Ismail. Private cancer centers include Institut Kanser MAKNA-Pusat Perubatan Universiti Kebangsaan Malaysia, Nilai Cancer Institute, Hospital Mutiara Penang, Sabah Medical Centre, Damansara Specialist Hospital, Pantai Medical Centre, and Gleneagles Medical Centre, no name a few. In this era of globalization, cancer patients also have the choice of deciding to go abroad for cancer treatment. Cross border consultation and treatment remains the choice of those who could afford the cost.

\section{Choice of treatment}

There are national guidelines for management of breast cancer (second edition, 2010) and for management of cervical cancer (2003) in order to guide healthcare providers on the recommended treatment for respective patients. These guidelines were produced by groups of experts from all over the country including oncologists, pathologists, surgeons, radiologists and other clinical specialists. Treatment of choice depends on the types of cancers and individual patients' conditions as provided in the guidelines. The treatments available include surgery, chemotherapy, radiotherapy, brachytherapy, medication therapy and palliative care. There may be combined therapy as well. As an example, for early stage breast cancer, surgical treatment is combined with chemotherapy as an added treatment to improve the outcome and cure rates. For late stage cancer, chemotherapy is used to control the growth and to downsize the tumor. The use 
of chemotherapy may also help to control symptoms and improve survival. As for colorectal cancer, the treatment is individualized according to patient's conditions based upon a number of factors including the anatomical location of the tumor, biochemical and genetic markers, and response to systemic therapy preoperatively.

\section{Anti-cancer drugs}

A variety of anti-cancer drugs are available and used as recommended by national and international guidelines on cancer treatments. However, the data from the Malaysian Statistics on Medicines 2005 report showed that the utilization of anti-cancer drugs in the Malaysian population do not even rank among the top 30 by utilization level or by $\operatorname{cost}^{18)}$.

\section{Prognosis of cancer patients}

\section{Five year survival rate}

A study of newly diagnosed breast cancer patients in University of Malaya Medical Centre in Kuala Lumpur diagnosed in two times periods of 1993 to 1997, and 1998 to 2002 reported that there was improvement in 5 -year observed survival of $58.4 \%$. The improvement in survival was significantly seen in all co-variates except for those aged 40 years and below, tumor size 2 to $5 \mathrm{~cm}$, grade 3 and patients with Stage IV disease ${ }^{19)}$. The information on survival trends for other types of cancers is rather limited.

\section{Support for cancer patients}

\section{Support group}

There exist many support groups for cancer patients in Malaysia. One of the most striking figure is the National Cancer Council or Majlis Kanser Nasional (MAKNA) which was established in 1994. This not-for-profit organization functions as a body that provides curative, preventive, research and support services to cancer patients, their families, high-risk groups and the general public in Malaysia. They also provide cancer awareness and education programs to educate the public. Other support groups include the National Cancer Society Malaysia, Malaysian Oncological Society, and few other regionally established cancer support groups.

\section{Financial support}

MAKNA has launched a few programs to help raise funds for cancer patients. The programs mostly raise funds through voluntary contributions from the public. The programs are the "Direct Debit Donor Program", "Loose Change Campaign", "Payroll Giving" (monthly fixed donation from payroll) and "Sen Save Lives" (collecting loose change at high traffic locations throughout the country). Under the MAKNA's Bursary Program, this organization then provides monetary assistance for financially challenged cancer patients requiring support for treatment costs.

\section{Public education and campaign}

As noted earlier, despite the existence of effective screening as preventive measure against cervical cancer, the uptake of Pap smear is poor. Lack of knowledge about cancer and social factors are the most common reasons why many women avoid this screening test though it is known to be life-saving. In response, there have been numerous initiatives by the Ministry of Health and non-governmental organizations such as MAKNA and The National Cancer Society Malaysia to encourage women to go for regular Pap smears.

\section{The way forward}

\section{National Cancer Patient Registry}

In 2007, The National Cancer Patient Registry (NCPR) was proposed as the first database to record detailed information on cancer patients in Malaysia, unlike the NCR which reported only on the incidence of cancers. It was due to the realization that although there have been improvements in cancer treatment, new therapies may potentially cause an exponential increase in the cost of cancer treatments. Therefore, justification for the use of these treatments is mandated.

The main objectives of the NCPR include determining disease burden attributable to cancer by quantifying the magnitude of cancer morbidity and mortality, and its geographic and temporal trends; describe the natural history of cancers; evaluate cancer treatment to determine their effectiveness; monitor the safety of products and services used in the treatment of cancers; and evaluate access to and quality of treatment services. It is hoped this project will help support clinical decision making and local policy development, as well as improving access to treatment and delivery of cancer services in Malaysia. The data collection would be from all patients with confirmed diagnosis of cancer at participating sites. The observational cohort study began in early 2008 and is anticipated to be completed after 10 years ${ }^{20)}$.

\section{Cancer research}

Cancer research in Malaysia is also enhanced with the establishment of the MAKNA-UPM Cancer Research Laboratory (MATCH), an alliance between MAKNA and the Institute of Bioscience of Universiti Putra Malaysia. They have successfully produced some remarkable results, one of which confirmed the efficacies of the medicinal Zingiber zerumbet as an anti-cancer compound. It was found that this agent could successfully suppress the proliferation of human cervical car- 
cinoma cell lines (HeLa), and the progression of tumor in mice induced with cervical cancer. When combined with Cisplastin (a platinum-based chemotherapy drug) as a co-treatment for the mice, the treatment showed some positive effects. Two other studies were also done by the same group of MATCH researchers on anti-cancer properties of plant-derived and chemically synthesized compounds on breast cancer cells, and anti-tumorogenic effects seen in female mice ${ }^{21)}$.

\section{Future consideration for screening}

A study done in two major teaching hospitals in Malaysia showed that out of 84 breast cancer tissue samples, human mammaglobin (hMAG) which is a secreted protein used as a specific marker for breast cancer has been detected in epithelial cells of mammary glands of the breast. It was expressed in 50 samples (59.6\%) and increased with cancer grade. Thus hMAG may serve in the diagnosis and the assessment of progression of breast cancer especially in more advanced cancer ${ }^{22}$.

\section{Conclusion}

Cancer is one of the debilitating health threats for women of Malaysia. Of the 10 most common cancers, breast cancer has the highest incidence, followed by colorectal cancer and cervical cancer. There is still room for reducing the incidence of cancer through public awareness program and screening. However, it is good to know that Malaysia offers a variety of choice of treatments; guided by the availability of national guidelines, such as that for breast and cervical cancers. Research has been conducted actively to find out the best future approach on screening, diagnosis and treatment for cancers affecting women. This is made possible with the government taking the lead and the important works which are highly supported by relevant non-governmental bodies and the public. It is hoped that the recently established NCPR will provide better understanding of the situation of cancers affecting the population in general, as well as those affecting women in Malaysia.

\section{Acknowledgment}

This work was supported in part by a non-profit organization "Epidemiological and Clinical Research Information Network (ECRIN)".

\section{References}

1) Department of Statistics, Malaysia. Distribution of population and sex ratio by sex and state, 2010. Available at: http://www.statistics. gov.my/portal/images/stories/files/LatestReleases/banci/jadual2. pdf [date accessed 10 June 2011].

2) Zainal Ariffin O, Zainuddin MA, Nor Saleha IT. Malaysian Cancer Statistics - Data and figure, Peninsular Malaysia 2006. National Cancer Registry, Ministry of Health Malaysia.

3) Lim GCC, Halimah Y. Lim TO. The first report of the National Cancer Registry: Cancer incidence in Malaysia 2002. National Cancer Registry, Ministry of Health Malaysia.
4) Lim GCC, Halimah Y. The second report of the National Cancer Registry. Cancer Incidence in Malaysia 2003. National Cancer Registry, Ministry of Health Malaysia.

5) Lim GCC, Sanjay R, Halimah Y. Cancer incidence in Peninsular Malaysia, 2003-2005. National Cancer Registry, Ministry of Health Malaysia.

6) Yip CH, Mohd Taib NA, Mohamed I. Epidemiology of breast cancer in Malaysia. Asian Pac J Cancer Prev. 2006; 7:369-74.

7) X. Castlellsagué, S. de Sanjosé, T. Aguado, K.S. Louie, L. Bruni, J. Muñoz, M. Diaz, K. Irwin, M. Gacic, O. Beauvais, G. Albero, E. Ferrer, S. Byrne, F.X. Bosch. HPV and cervical cancer in the world 2007 report. 2007; 25, 3.

8) Yip, C.H., \& Abdullah, N.H. (2003) Spectrum of breast cancer in Malaysian Women: Overview. World Journal of Surgery 27, 921923.

9) Ismail MN, Chee SS, Nawawi H, Yusoff K, Lim TO, James WPT. Obesity in Malaysia. The International Association for the Study of Obesity. Obesity reviews. 2002; 3:203-208.

10) Toh GT, Kang P, Lee SSW, Lee DS-C, Lee SY, et al. BRCA1 and BRCA2 Germline Mutations in Malaysian Women with EarlyOnset Breast Cancer without a Family History. PLoS ONE. 2008; 3(4): e2024.

11) Naidu R, Har YC, Taib NAM. Genetic polymorphisms of TP53binding protein 1 (TP53BP1) gene and association with breast cancer risk. APMIS. 2011; 119(7):460-7.

12) Loh SY, Chew SL. Awareness and practice of breast self examination among Malaysian women with breast cancer. Asian Pac J Cancer Prev. 2011; 12(1):199-202.

13) Ministry of Health, Malaysia. Clinical Practice Guidelines: Management of Breast Cancer. MOH/P/PAK/212.10 (GU), November 2010.

14) Domingo EJ, Noviani R, Noor MR, Ngelangel CA, Limpaphayom KK, Thuan TV, Louie KS, Quinn MA. Epidemiology and Prevention of Cervical Cancer in Indonesia, Malaysia, the Philippines, Thailand and Vietnam. Vaccine. 2008; 26(12):71-9.

15) Nor Hayati Othman, Beena C R Devi, Y Halimah. Cervical cancer screening: patients understanding in major hospitals in Malaysia. Asian Pacific journal of cancer prevention. 10(4):569-74.

16) Dunn RA, Tan AK. Cervical cancer screening in Malaysia: Are targeted interventions necessary? Soc Sci Med. 2010; 71(6):108993.

17) $\mathrm{WHO} / \mathrm{ICO}$ Information Centre on HPV and Cervical Cancer (HPV Information Centre). Human Papillomavirus and Related Cancers in Malaysia. Summary Report 2010. Available at www. who. int/ hpvcentre.

18) Sameerah SAR, Sarojini S. Malaysian Statistics on Medicine 2005. Available at http://www.crc.gov.my/nmus.

19) Nur Aishah Taib, MN Akmal, I Mohamed, Cheng-Har Yip. Improvement in Survival of Breast Cancer Patients - Trends in Survival over Two Time Periods in a Single Institution in an Asia Pacific Country, Malaysia. Asian Pacific J Cancer Prev. 2011; 12 : 345-349.

20) GCC Lim, D Azura. National Cancer Patient Registry: A Patient Registry/ Clinical Database to Evaluate the Health Outcomes of Patients Undergoing Treatment for Cancers in Malaysia. Med J Malaysia. 2008; 63(C).

21) Dr Ahmad Bustamam $\mathrm{Hj}$ Abdul. 1. Anti-cancer Effects of Zerumbone for Zingiber Zerumber on Human Cervical Cancer Cells (HeLa) and Female Balb/c mice Induced with Cervical Cancer. 2. Anti-cancer Properties of Plant-derived and Chemically Synthesized Compounds on Breast Cancer Cells 3. Antitumourigenic Effects of Corresponding Chemical Analogues in Chemical Intraepithelial Neoplasia of Female Balb/c Mice. MAKNA Annual Scientific Report, 2007. Available at http://www. makna.org.my/annualreport07/dr-bustaman.html.

22) Al-Joudi FS, Kaid FA, Ishak I, Mohamed N, Osman K, Alias IZ. Expression of human mammaglobin and clinicopathologic correlations in breast cancer: The findings in Malaysia. Indian J Pathol Microbiol. 2011; 54:284-9. 